\title{
Attitude Determination for the Pico-Satellite UWE-2
}

\author{
Marco Schmidt, Karthik Ravandoor, Oliver Kurz, Stephan Busch, Klaus Schilling \\ University Wuerzburg, Informatik VII: Robotics and Telematics \\ D-97074 Wuerzburg, Germany \\ (Tel.+49-931-8886647,e-mailschi@informatik.uni-wuerzburg.de)
}

\begin{abstract}
Modern miniaturization technologies enable the realization of very small satellites at masses below $1 \mathrm{~kg}$, nevertheless limited attitude determination and actuator performances raise challenging control problems. Specific attitude determination aspects discussed at the example of UWE (University of Wuerzburg Experimental satellite), a standardized pico-satellite platform related to technology demonstration and space research. UWE-1 is in orbit since October 2005 and successfully completed its mission on telecommunication experiments. The successor satellite UWE-2 is focusing on an advanced Attitude Determination System (ADS) based on miniature sensors. This paper will address implementation details for the ADS, presenting in particular results from the performance evaluations in simulations and tests.
\end{abstract}

\section{INTRODUCTION}

Miniaturization technologies significantly impact satellite system design approaches. While in the traditional way single, high-performance satellite platforms of several tons were designed, now distributed swarms of small satellites are intensively discussed (Ankersen, 2008). Obvious promises are improved spatial and temporal coverage in Earth observation, as well as increased robustness. Nevertheless, technology challenges related to attitude and orbit determination and control (Schilling and Fullmer, 2003), as well as to satellite coordination on basis of the sensor and telecommunication infrastructure are to be solved.

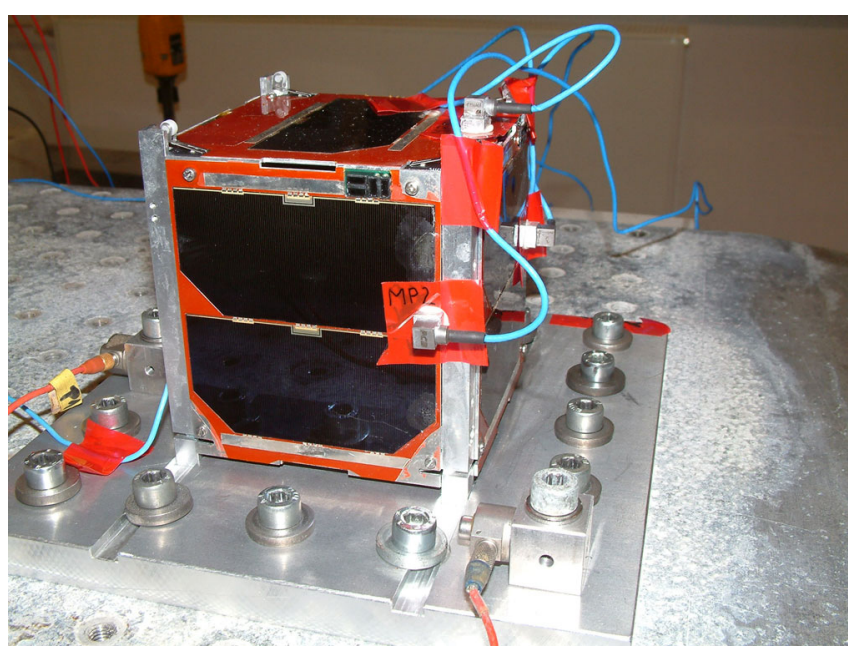

Fig. 1. The pico-satellite UWE-2 during vibration test.

For this ambitious goal, the University Wuerzburg established a road map for technology implementations leading towards the realization of coordinated swarms of pico-satellites. The UWE-1 satellite (University Wuerzburg's Experimental Satellite) (Barza et al., 2006, Schilling et al., 2005) was the first German pico-satellite, launched in 2005. It successfully completed telecommunication experiments related to optimization of parameters in the Internet Protocol (IP) in space environment (Schmidt et al., 2006, Zeiger et al. 2006), exhibiting significant delays and noise levels. The successor UWE-2 (c.f. Fig. 1) was developed in 2007 and has the objective to demonstrate the capabilities of extremely small satellites in the field of attitude determination.

In this paper the basic architecture of UWE-2 system is introduced in chapter 2. Chapter 3 addresses the sensor hardware employing magnetometers, sun sensors, gyros and accelerometers, while chapter 4 presents the algorithms to derive attitude determination. Because of the non-linearity of attitude dynamics and kinematics, an Extended Kalman Filter $(E K F)$ is used to fuse the gathered sensor data. Simulation results of this ADS system are summarized in chapter 5 .

\section{THE UWE-2 ELECTRICAL SYSTEM DESIGN}

The UWE-2 mission emphasizes technology tests on accurate attitude determination as a preparatory step for interpretation of remote observation data and for coordination of satellite swarms. The UWE platforms are an integral element in spacecraft system design education within the SpaceMaster program (www.spacemaster.uni-wuerzburg.de) (Schilling, 2006). The design follows the CubeSat standards (Twiggs, 2003) in order to enable implementation of such a picosatellite with a mass of about $1 \mathrm{~kg}$ within less than one year. It includes all standard subsystems (e.g. power, on board data handling, telecommunication), but was optimized with respect to small size and low weight. The satellite, together with its electronic boards (c.f. Fig. 2), was designed, developed and manufactured at the University Wuerzburg. 


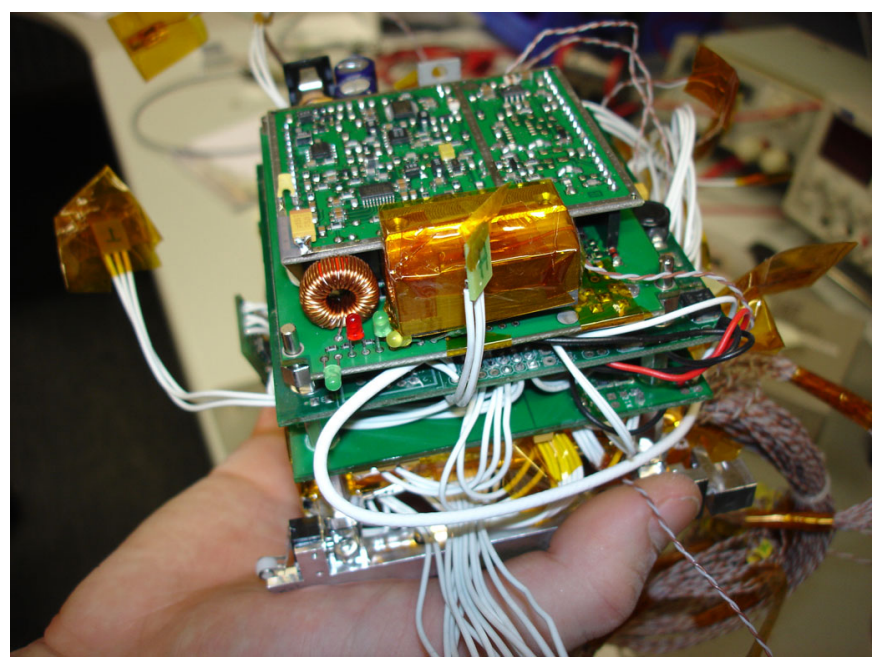

Fig. 2. The highly integrated electronic boards inside UWE-2

Special attention was paid to the low power consumption requirements, leading to the selection of the Hitachi H8 microprocessor. It is capable of running a complex operating system, providing access to a broad spectrum of applications and protocols. UWE uses the uClinux operating system (a GNU/Linux based operating system) for On Board Data Handling (OBDH).

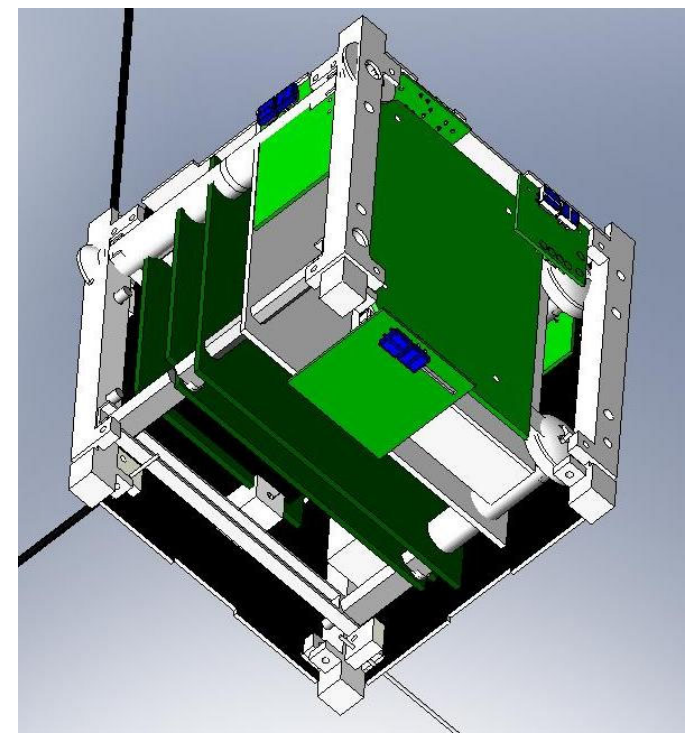

Fig. 3. Structure layout of the UWE-2 satellite

The power is generated from highly efficient triple-junction GaAs solar cells, providing an efficiency of $28 \%$. The Communication subsystem is based on a transceiver using amateur frequency bands (VHF/UHF).

The first UWE satellite platform was launched in October 2005 by a Cosmos-3 rocket (Schilling et al. 2005), based on a similar electrical configuration. For UWE-2 main emphasis was on the sensor suite for position and attitude determination, including a GPS. A major simplification concerns attitude control, where only stabilisation by permanent magnets is provided, which align the satellite to the corresponding magnetic field of Earth.

\section{THE HARDWARE OF THE ATTITUDE DETERMINATION SYSTEM}

Due to the limited dimensions of the satellite it is not possible to integrate highly accurate attitude determination sensors, like star trackers or horizon detectors, because of their volume and power consumption needs. Nevertheless, position and attitude determination are required basics, before measurements can be further processed and interpreted. To enable an appropriate attitude determination on UWE-2, the pico-satellite was equipped with redundant sensors for robust and accurate measurements. The sensor equipment is mainly based on commercial micro-electromechanical system (MEMS) components, which were selected with respect to low mass and power consumption. Complete 3-axis information can neither be provided by magnetometers nor by sun sensors. Therefore a combination of both is to be used to derive solutions of the equations for attitude determination. The magnetometers contribute measurements of the magnetic field vector induced by the Earth's magnetic field on the satellite body. The sun sensors detect the sun incidence angle to the satellite's reference frame.

The UWE-2 satellite carries six pairs of perpendicularly mounted individual sun sensors, one on each panel. Therefore the Sun will be continuously in the field of view, when the satellite is not in eclipse. For this situation additional sensors must be foreseen to guarantee complete attitude coverage. For complementary measurements three miniature gyros are included, contributing changes in attitude and a direct measurement of turn rates. They are not influenced, due to the employed physical effect, by external electromagnetic fields. Therefore, they offer the possibility to provide turn rate information at a faster rate and with a higher reliability than absolute sensors with no dependence on further prerequisites. An accelerometer complements the gyros to form the inertial navigation system. Two-lineelement (TLE) data can - to some extent - provide ground measurements of the absolute position, such that an off-line correction of the relative data on ground is possible.

On-board the absolute position of UWE-2 is determined by a GPS receiver. It provides position data at a higher accuracy compared to the accelerometer/TLE solution. A specialized Phoenix GPS receiver (Montenbruck et al., 2008) was integrated in UWE-2 providing accurate time and position information. In orbit tests will demonstrate the performance of this important component of the ADS.

In general, sensors with digital output signal and included signal conditioning are preferred. This way the very tedious task of tuning the systems parameters is simplified such that more emphasis can be placed on improving the information quality in the processing step. A digital sensor is more robust to noise, as all analogue circuit parts are integrated in the sensor chip and therefore analogue lines are minimized in length.

To allow consistent implementation methods for every sensor, the Serial Peripheral Interface (SPI) bus system was selected as underlying physical communication layer. This 
master-slave bus provides very high transmission rates with low protocol overhead and easy implementation in the software controlling part.

For simple realisation of the integrated hardware and software, as well to optimum provision of computing power for data pre-processing, the microcontroller is used as key component of the attitude determination system. It provides the command link to trigger the sensor activities and sends the collected data to the OBDH.

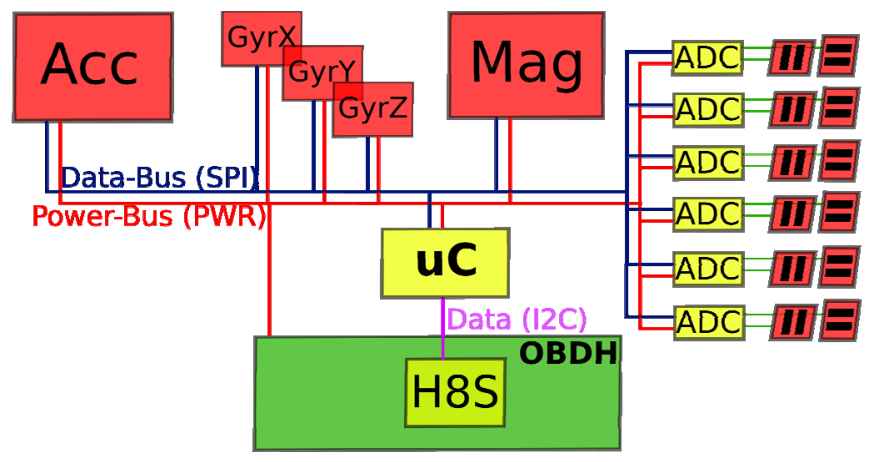

Fig. 4. Schematic of the data flow between the ADS sensor system and the OBDH

\section{ALGORITHMS OF THE ATTITUDE DETERMINATION SYSTEM}

The attitude determination system of UWE comprises of the orbit generation subsystem, subsystem for obtaining the reference sun vector and magnetic field vector and an Extended Kalman Filter (EKF), performing the data fusion and estimating the satellite's attitude. Figure 5 depicts the complete functional block diagram of the attitude determination system.

The orbit generator relies on the Two Line Element (TLE) set for the initial orbit elements required to generate the orbit positions. Since TLE data are generated in a specific way, the orbit elements are reconstructed by calculating the osculating values using the Simplified General Perturbations (SGP4) propagation model (Hoots et al., 1988). Although the SGP4 model can be used for successive position generation, it is computationally more demanding compared to a simplified orbit generator which generates the orbit positions by solving Kepler's equation.

The orbit generator uses the general perturbations method to predict variations in the orbital parameters due to noise effects from the environment. The perturbations considered for UWE's orbit generator are third-body perturbations due to gravitational forces of the Sun and the Moon, perturbations due to non homogeneous gravity potential of the Earth and perturbations due to atmospheric drag.

The SGP4 model was used as benchmark to compare the accuracy of position generation. Over a period of time the simplified orbit generator accumulates errors and needs a recalibration. To check the error growth the SGP4 model was used periodically to get the expected orbital parameters and use them to feed the simplified orbit generator, thus resetting the errors accumulated. The position errors remained within $20 \mathrm{~km}$ of the value predicted by SGP4 compared to an error of $100 \mathrm{~km}$ without error resetting.

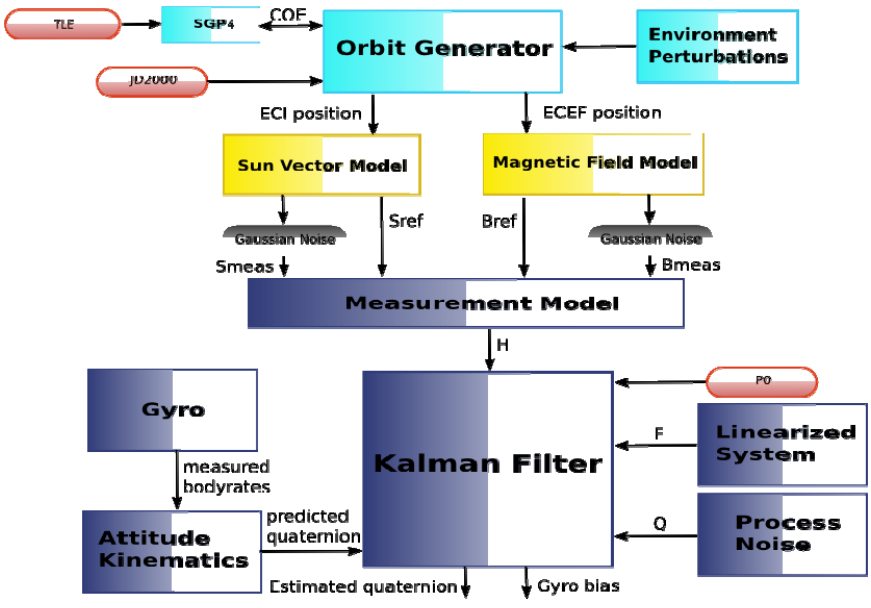

Fig. 5. Functional block diagram of the UWE-2 attitude determination system

The attitude estimator requires a reference magnetic field vector and sun vector information to compare the information provided by the magnetometer and the sun sensors. Due to constraints of storage space on board the satellite computer, storing large amount data for expected magnetic field values is not feasible. Instead, using the satellite positions provided by the orbit generator, the reference magnetic field value is estimated by using the International Geomagnetic Reference Field (IGRF) 2005 standard, mathematical model. The reference value obtained is defined in the Earth Centered Earth Fixed (ECEF) reference frame. Similarly for the reference sun vector value, a sun vector model (Shuster and Oh, 1981) with Earth as the observer at the center of the celestial sphere and Sun in it's ecliptic around the Earth is used to predict the sun vector in the Earth Centered Inertial (ECI) frame. The reference vectors are transformed into orbit frame using the orbital elements and finally into the body frame using the estimated attitude.

The attitude of the satellite is defined with respect to the orbit frame whose origin coincides with the geometrical center of the satellite. The center of mass and the geometrical center of the satellite are assumed to coincide. The satellite's RollPitch-Yaw frame rotates relative to the inertial frame. The attitude parameterization for UWE is done using the quaternions. Quaternion representation of rotation leads to convenient representation of attitude kinematics as linear equations. Additionally quaternion unit norm provides an easy way to preserve the orthogonality of the rotation matrix. Further, the composite rotations can be performed in terms of quaternion multiplication without involving trigonometric functions.

At the heart of UWE's attitude estimation algorithm is the extended Kalman filter (EKF). The nonlinear state equations are linearized about the best estimate using the EKF. While the attitude kinematics are being estimated by the filter, the 
satellite dynamics are measured using the onboard gyro. However, the gyro suffers from drift errors. Use of gyro data to propagate the attitude kinematics leads to inaccurate attitude estimates. Thus the body rates measured by the gyros need corrections. The satellite's angular velocity can related to gyro output as

$$
\omega(t)=\omega_{m}(t)-b-n_{r}(t)
$$

where $b$ is the drift rate bias, $\omega_{r}$ is the gyro output and $n_{r}$ drift rate noise, which is assumed to be a Gaussian whitenoise process. The drift rate bias $b$ itself is driven by a second Gaussian white-noise process, the gyro drift-rate ramp noise. Thus the state space model (Lefferts et al., 1982) is

$$
\begin{gathered}
\dot{q}=\frac{1}{2} \Omega\left(\omega_{m}-b-n_{r}\right) q \\
\dot{b}=n_{w}(t)
\end{gathered}
$$

The error state model for the EKF is obtained using a small angle approximation. Thus the EKF estimates error signals for the rotation angles and drift rate bias using the error state model (Lefferts et al., 1982, Mourikis et al., 2007)

$$
\begin{gathered}
{\left[\begin{array}{c}
\delta \dot{\theta} \\
\Delta \dot{b}
\end{array}\right]=\left[\begin{array}{cc}
-[\hat{\omega} \times] & -I_{3 \times 3} \\
0_{3 \times 3} & 0_{3 \times 3}
\end{array}\right]\left[\begin{array}{c}
\delta \theta \\
\Delta b
\end{array}\right]+\left[\begin{array}{cc}
-I_{3 \times 3} & 0_{3 \times 3} \\
0_{3 \times 3} & 0_{3 \times 3}
\end{array}\right]\left[\begin{array}{l}
n_{r} \\
n_{w}
\end{array}\right]} \\
\delta \dot{x}=F_{c} \delta x+G_{c} n
\end{gathered}
$$

The process-noise covariance matrix of the noise vector $n$ is given by

$$
Q_{c}=\left[\begin{array}{ll}
\sigma_{r}^{2} I_{3 \times \mathbf{3}} & 0_{3 \times \mathbf{3}} \\
0_{3 \times 3} & \sigma_{w}{ }^{2} I_{3 \times \mathbf{3}}
\end{array}\right]
$$

In order to predict the quaternion either a zeroth order or a first order numerical integrator can be used. This reduces the computational load compared to using the $4^{\text {th }}$ order RungeKutta integration method. If the body rate vector $\omega(t)=\omega$ is constant over the integration period $\Delta t$ then the zeroth order quaternion integrator can be used to predict the quaternion according to

$$
\begin{aligned}
\bar{q}\left(t_{k=1}\right)= & \left.\cos \left(\frac{|\omega|}{2} \Delta t\right) I_{\mathbf{4} \times \mathbf{4}}\right) \hat{q}\left(t_{k}\right) \\
& +\left(\frac{1}{|\omega|} \sin \left(\frac{|\omega|}{2} \Delta t\right) \Omega(\omega)\right) \hat{q}\left(t_{k}\right)
\end{aligned}
$$

If the body rates $\omega(t)$ vary linearly over the integration interval, then the first order integrator can be used for quaternion prediction according to

$$
\begin{aligned}
& \bar{q}\left(t_{k+1}\right)=\left(\exp \left(\frac{1}{2} \Omega\left(\omega_{a}\right) \Delta t\right)\right) \hat{q}\left(t_{k}\right) \\
& +\left(\frac{1}{48}\left(\Omega\left(\omega_{k+1}\right) \Omega\left(\omega_{k}\right)-\Omega\left(\omega_{k}\right) \Omega\left(\omega_{k+1}\right)\right) \Delta t^{2}\right) \hat{q}\left(t_{k}\right)
\end{aligned}
$$

Using the discrete gyroscope model, the estimated values of the body rate and bias can be written as

$$
\begin{gathered}
\hat{\omega}_{k \mid k-1}=\omega_{m}-\bar{b}_{k \mid k-1} \\
\bar{b}_{k \mid k-1}=\hat{b}_{k-1 \mid k-1}
\end{gathered}
$$

After time updates and the predictions of the error signals are made by the EKF, next the measurement updates are carried out.

Further, the Kalman gain is given by

$$
K_{k}=M_{k} H^{T}\left(H M_{k} H^{T}+R\right)^{-1}
$$

where $M_{k}$ is the predicted error covariance, $H$ is the measurement matrix and $R$ measurement error covariance matrix. $H$ is computed (Psiaki, 1989) as

$$
H=\left[\begin{array}{ll}
\frac{-2 \hat{B}_{\text {ref }} B_{\text {meas }}^{T}}{\left\|B_{\text {meas }}\right\|\left\|\hat{B}_{\text {ref }}\right\|} & 0_{\mathbf{3} \times \mathbf{3}} \\
\frac{-2 \hat{S}_{\text {ref }} S_{\text {meas }}^{T}}{\left\|S_{\text {meas }}\right\|\left\|\hat{S}_{\text {ref }}\right\|} & 0_{\mathbf{3} \times \mathbf{3}}
\end{array}\right]
$$

and the measurement error covariance matrix is a diagonal matrix, whose elements are the variance of the three axes of the magnetometer and sun sensor. Finally the error states are updated by

$$
\Delta \hat{x}_{k}=K_{k} v
$$

where $v=\left[z_{k}-h(\hat{x})\right]$ is the innovation or the residual of the filter. The EKF used in UWE-2 is multiplicative. This implies that the estimated attitude error signal represents a small rotation from the predicted to the expected attitude (Bak, 1999, Mourikis et al., 2007). Thus the estimated attitude is obtained using

$$
\hat{q}_{k \mid k}=\left[\frac{\frac{1}{2} \Delta \hat{\theta}_{k}}{\sqrt{1-\frac{1}{4} \Delta \hat{\theta}_{k}{ }^{T} \Delta \hat{\theta}_{k}}}\right] \bar{q}_{k \mid k-1}
$$

However, the state update method for gyro drift bias is additive and is of the form

$$
\hat{b}_{k \mid k}=\bar{b}_{k \mid k-1}+\Delta \hat{b}_{k}
$$

Finally, the estimation error covariance is updated

$$
P_{k}=\left(I-K_{k} H\right) M_{k}
$$

To start the attitude estimation, a good guess of the initial attitude is needed. For this purpose the simple approach described by the TRIAD algorithm (Shuster and Oh, 1981) is used. The TRIAD algorithm is a deterministic, non optimal solution based on two vector observations. If $V_{1}$ and $V_{2}$ are two nonparallel observed unit vectors using the magnetometer and sun sensors in the body frame and $W_{1}$ and $W_{2}$ are 
the corresponding reference unit vectors, then the attitude $A$ satisfies the condition

$$
A V_{1}=W_{1} \text { and } A V_{2}=W_{2}
$$

Once the initial attitude information $A_{3 \times 3}$ is determined, the quaternion components are extracted by using

$$
A(q)=\left(q_{4}^{2}-q^{2}\right) 1+2 q q^{T}-2 q_{4} Q
$$

Where $Q=Q\left(q_{1}, q_{2}, q_{3}\right)$ is the symmetric skew matrix.

The TRIAD covariance matrix is a body-referenced covariance matrix. The Cartesian error covariance matrix of error angles can be expressed in terms of the observation vectors using

$$
\begin{gathered}
\bar{P}_{\theta \theta}=\sigma_{1}^{2} I+\frac{1}{\left|W_{\mathbf{1}} \times W_{2}\right|}\left(\left(\sigma_{1}^{2}-\sigma_{2}^{2}\right) W_{\mathbf{1}} W_{1}^{T}\right) \\
+\frac{1}{\left|W_{\mathbf{1}} \times W_{\mathbf{2}}\right|}\left(\sigma_{1}^{2}\left(W_{\mathbf{1}} \cdot W_{\mathbf{2}}\right)\left(W_{\mathbf{1}} W_{\mathbf{2}}^{T}+W_{\mathbf{2}} W_{1}^{T}\right)\right) \\
\text { where } \sigma_{1}^{2}=\sigma_{v 1}^{2}+\sigma_{w 1}^{2} \text { and } \sigma_{2}^{2}=\sigma_{v 2}^{2}+\sigma_{w 2}^{2}
\end{gathered}
$$

\section{SIMULATION RESULTS}

This attitude estimator was tested in simulations to characzterize its performance. The simulation uses sample TLEs of a satellite in Low Earth Orbit (LEO). The orbital elements from the epoch time when TLE set was generated were propagated up to the start of simulation and then used for simulation. Simulation is carried out for a duration of 2 orbits. Estimations are done every 5 seconds. The time interval chosen is such that the variations in the satellite body rates are almost linear in order to use the first order quaternion integrator. Through adaptive techniques a suitable amount of measurement noise was added to account for the errors in modelling the sensors. This way a balance between emphasis on the system process model and the measurement model is achieved. The simulation is assuming the situation that for both, sun sensor and magnetometer, the sensor data are reliably available all the time. As seen from the simulation, the estimated signal is able to track the expected signal satisfactorily while keeping the estimation error well within the deviation limits.

Results from the simulation are presented here. The quaternion estimation error in different components are found to be $\pm 0.5 \%$ for Q1 , $\pm 15.7 \%$ for Q2 , $\pm 6.32 \%$ for Q3 and $\pm 5.9 \%$ for Q4 component.In addition the mean error in body angles in terms roll, pitch and yaw are found to be maximum of $\pm 21.32^{\circ}$ in roll angle, $\pm 19.92^{\circ}$ in pitch angle and $\pm 11.05^{\circ}$ in yaw angle.

Salient features of the UWE-2 ADS include:

- The hybrid orbit generator, a combination of SGP4 and simplified orbit generator, regulates the error growth in position prediction. Since the reference models used for deriving the reference magnetic and sun vector are functions of the satellite's position, the improved position generation has direct impact on the quality of the attitude estimation.

- Use of onboard gyros avoids the need to estimate attitude dynamics by modelling the complex dynamic model. This leads to improved attitude kinematics prediction

- Use of TLE for initial orbital parameters and TRIAD algorithm for initial state values for the Kalman filter operation makes the whole process of attitude estimation closed and independent.

- The filter innovation $v$ and the measurement matrix $H$ consistent with the innovation are different from the conventional filter practice. These changes handle the nonlinearities in a manner better than brute force normalization (Psiaki, 1989).

- Forced normalization of quaternion error signals (Deutschmann et al., 1992) is done to expedite the error convergence as the normalization technique is found superior to tedious and unreliable filter tuning.
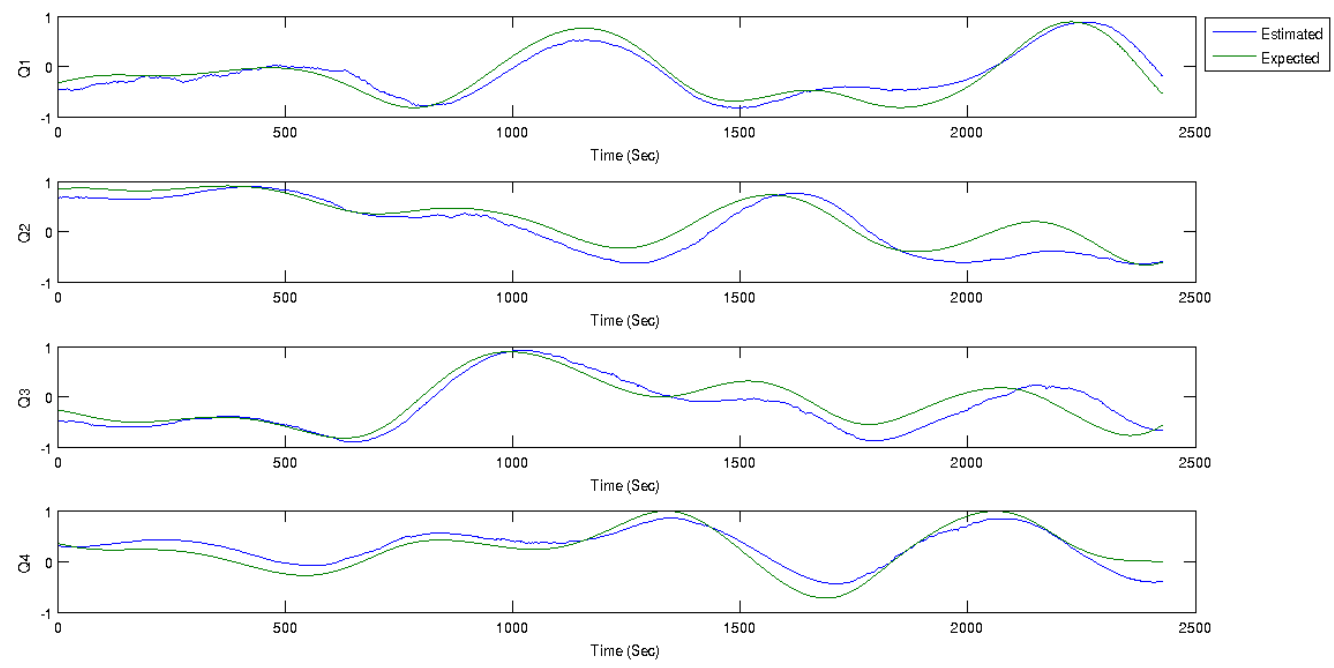

Fig. 6: Expected and estimated quaternion signals 

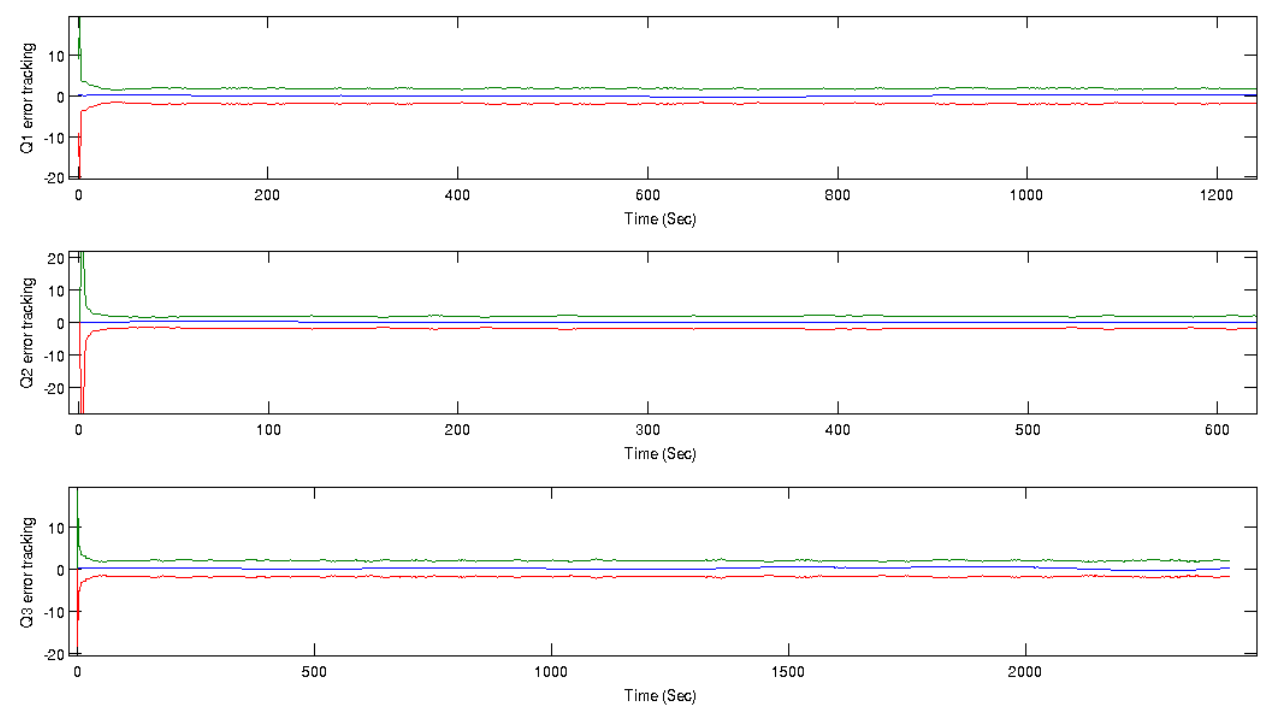

Fig. 7: Estimation error for vector components of the quaternion

- For lean operations on board the satellite, zeroth and first order quaternion integrators are used for predicting the quaternion during time update phase of the EKF.

- Use of multiple type of sensors to update the predictions, gives a better possibility to support measurement update of the EKF when data from one type of sensor is notreliable. In the absence of data from any sensor to perform the measurement update, the attitude estimation can still be performed by using the gyro alone.

\section{CONCLUSIONS}

Attitude determination for pico-satellites faces challenges due to the limitations in volume and mass for related sensors. A sensor concept based on sun sensors and magnetometers, complemented by a GPS is presented. Using an extended Kalman filter in combination with orbit predictors, an appropriate performance of the attitude determination system can be achieved as demonstrated in simulations. When these results will be proved in orbit, too, this will provide a basis for coordinated operations of satellite swarms, promising interesting capabilities in telecommunication and earth observation.

\section{REFERENCES}

Ankersen, F. (ed.) (2008), Proceedings 3rd International Symposium on Formation Flying, Missions and Technologies, ESA SP-654, Noordwijk

Bak, T. (1999), Spacecraft Attitude Determination- a Magnetometer Approach. $\mathrm{PhD}$ thesis, Aalborg University.

Barza, R., Y. Aoki, K. Schilling (2006), Cubesat UWE-1 Technology Tests and in Orbit Results. In: 57th International Astronautical Congress, IAC-06-B5.3.07

Deutschmann, J., I. Bar-Itzhack, and K. Galal (1992), Quaternion Normalization in Spacecraft Attitude Determination. In: AIAA/AAS Astrodynamics Conference

Hoots, F. R., R. L. Roehrich, and T.S. Kelso (1988), Spacetrack report No. 3, Models for Propagation of NORAD Element Sets
Lefferts, E.J., F.L. Markley, and M.D.Shuster (1982), Kalman Filtering for Spacecraft Attitude Estimation. Journal of Guidance 5, 417-429

Montenbruck, O., E. Gill, M. Markgraf (2006), Phoenix-XNS - A Miniature Real-Time Navigation System for LEO Satellites, Proceedings NAVITEC'2006, Noordwijk

Mourikis, A., N. Trawny, S. I. Roumeliotis, D. M. Helmick, and L. Matthies (2007), Autonomous Stair Climbing for Tracked Robots, The International Journal of Robotics Research 26, 737-758

Psiaki, M. L. (1989), Three-Axis Attitude Determination via Kalman Filter of Magnetometer Data, Guidance 13, 506-514

Schilling, K., R. Fullmer (2003), Control Approaches to Small Student Build Satellites, 5th International ESA Conference on Spacecraft Guidance, Navigation and Control Systems, ESA SP-516, Noordwijk, p. 483 -487.

Schilling, K., R. Barza, D. Ziegler (2005), UWE-1, der erste deutsche Pico-Satellit, Raumfahrt Concret 4/5, p. 45-49

Schilling, K. (2006), Design of Pico-Satellites for Education in Systems Engineering. IEEE Aerospace and Electronic Systems Magazine 21, p. 9-14

Schmidt, M., F. Zeiger, K. Schilling (2006), Design and Implementation of In-Orbit Experiments on the PicoSatellite UWE-1, In: Proceedings 57th International Astronautical Congress, IAC-06- E2.1.07

Shuster, M. D. and S.D. Oh (1981), Three-Axis Attitude Determination From Vector Observations, Journal of Guidance and Control, 4, 70-77

Twiggs, R. (2003), The next Generation of Innovative Space Engineers: University Students are Now Getting a Taste of Space Experience Building, Launching and Operating their own Space Experiments with Low-Cost Picosatellites. Proceedings of the $5^{\text {th }}$ ESA International Conference on Spacecraft Guidance, Navigation and Control Systems, p. 409-422

Zeiger, F., M. Schmidt, K. Schilling (2006), A Flexible Extension for Pico-Satellite Communication Based on Orbit Operation Results of UWE-1, In : 57th International Astronautical Congress, IAC-06- B5.2.05 